\title{
DIVERSIDAD, COMPOSICIÓN FLORÍSTICA Y ENDEMISMOS EN LOS BOSQUES ESTACIONALMENTE SECOS ALTERADOS DEL DISTRITO DE JAÉN, PERÚ
}

\section{DIVERSITY, FLORISTIC COMPOSITION AND ENDEMISM IN SECONDARY TROPICAL SEASONALLY DRY FORESTS IN JAEN, PERU}

\author{
José Luis Marcelo-Peña ${ }^{1}$, Carlos Reynel-Rodríguez ${ }^{2}$, Percy Zevallos-Pollito ${ }^{3}$, Fernando Bulnes-Soriano ${ }^{4}$ y \\ Alonso Pérez-Ojeda del Arco
}

\begin{abstract}
Resumen
Mediante el establecimiento de 40 transectos siguiendo la metodología de Gentry, se realizó un análisis de la diversidad, endemismos, composición florística y complementariamente la estructura de la vegetación leñosa de bosques estacionalmente secos (BTES) alterados de cuatro sectores del distrito de Jaén. Las familias más abundantes son Boraginaceae, Leguminosae, Malvaceae y Cactaceae; los géneros más abundantes son Cordia, Tetrasida, Esenbeckia y Browningia. Las especies más importantes son Cordia iguaguana, Tetrasida chachapoyensis y Browningia altissima, las tres son endémicas restringidas a los bosques estacionalmente secos del Marañón. La diversidad vegetal es moderada. Sin embargo, los resultados confirman que la zona presenta valores sorprendentemente altos en endemismo en contraste con BTES interandinos y BTES orientales de nuestro país, además de los BTES del sur occidente del Ecuador. Por lo tanto, su conservación merece especial consideración.

Palabras claves: Diversidad, composición florística, endemismos, bosques estacionalmente secos, Cordia iguaguana, Tetrasida chachapoyensis, Browningia altissima, conservación, Jaén, Perú
\end{abstract}

\begin{abstract}
A set of transects was established following the Gentry methodology, for determining tree diversity and endemism levels, floristic composition and complementary vegetation structure information in four locations of secondary, Tropical Seasonally Dry Forests (TSDF) of Jaen, Dp. Cajamarca, Peru. The most abundant families are Boraginaceae, Leguminosae, Malvaceae and Cactaceae; the most abundant genera Cordia, Tetrasida, Esenbeckia y Browningia. The species found to be important are Cordia iguaguana, Tetrasida chachapoyensis y Browningia altissima, the three of them are endemics restricted to the TSDF of the Marañon valley in Peru. Despite the fact that the tree diversity level found is moderate, there are high levels of endemism, in contrast with other interandean and eastern areas in Peru and Ecuador; for this reason, the conservation of the area of study should be prioritized.

Key words: Tree diversity, floristic composition, endemism, Tropical Seasonally Dry Forests, Cordia iguaguana, Tetrasida chachapoyensis, Browningia altissima, conservatio, Jaen, Peru
\end{abstract}

\section{Introducción}

Los bosques secos representan el $42 \%$ de todos los bosques tropicales y subtropicales del mundo (Murphy \& Lugo, 1986; Miles et al., 2006). Se presentan desde el nivel del mar hasta los $1000 \mathrm{~m}$ de altitud, aunque en los valles andinos e interandinos del Perú llegan hasta los $2350 \mathrm{~m}$ en el valle de río Mantaro y en el valle de Apurímac hasta los 2400 (Weberbaur, 1945; LinaresPalomino, 2004). Los bosques secos neotropicales se distribuyen desde Mèxico hasta Argentina, Bolivia, Paraguay y Brasil. Pennington et al. (2000) los clasifica en nueve áreas: América Central y El Caribe, costa Caribeña de Colombia y Venezuela, valles interandinos colombianos, costa peruano-ecuatoriana, valles interandinos ecuatorianos, peruanos $y$ bolivianos, región boliviano chiquitana, núcleo de pie de monte, núcleo del Paraná y las caatingas en Brasil.
Según el Mapa Forestal del Perú (INRENA, 1995), el bosque seco abarca un área de $39451 \mathrm{~km}^{2}(3.07 \%$ de la superficie del territorio nacional), con mayor extensión en los departamentos de Lambayeque, Piura y Tumbes, pero, existen fragmentos $\mathrm{y}$ remanentes de bosque seco en todo el Perú (LinaresPalomino, 2006). Linares-Palomino (2004) clasificó los bosques secos tropicales estacionalmente secos (BTES) del Perú en tres subunidades biogeográficas: Subunidad de BTES Ecuatoriales, Subunidad de BTES interandinos (Sistema del río Huancabamba, Sistema del río Marañón, Sistema del río Mantaro, Sistema del río Apurímac y otros remanentes menores) y Subunidad de BTES orientales.

Los bosques estacionalmente secos han recibido relativamente poca atención por parte de conservacionistas y ecologistas comparados con los bosques lluviosos, a pesar de ser los más amenazados. 
La razón principal de la destrucción masiva de este ecosistema es la naturaleza fértil de su suelo, el cual es apreciado para la agricultura (Ratter et al., 1978). Su destrucción es exacerbada por las poblaciones humanas presentes en muchas zonas de vida de bosque seco neotropical (Murphy \& Lugo, 1995).

El BTES del sistema del Marañón ha cobrado notable importancia por su diversidad y por sus altos valores en endemismos. Sagastegui et al. (1999), manifiestan que los niveles de endemismo en el Norte del Perú (incluyendo los departamentos de Tumbes, Piura, Lambayeque, La Libertad, Amazonas y Cajamarca) son extremadamente altos, con más de 715 especies y 11 géneros reconocidos como endémicos. Hensold (1999), cita 318 taxones endémicos para el departamento de Cajamarca. De éstos, 53 taxones son de valles secos de la cuenca del Marañón y 15 taxones son de los valles estacionalmente secos de la vertiente oeste. Weigend (2002), afirma que los bosques estacionalmente secos de las vertientes de los Andes y los valles interandinos son menos conocidos, poseen igual diversidad $y$ hasta pueden superar el nivel de endemismos encontrados en los bosques húmedos.

Estudios recientes, desarrollados en los bosques estacionalmente secos del distrito de Jaén (Marcelo-Peña, 2007), reporta un total de 29 especies endémicas de ocho sectores en áreas no mayores de cuatro hectáreas cada una, y los considera como los bosques con valores más altos en endemismos para los BTES del norte del Perú y sur occidente de Ecuador. Sin embargo, aún queda mucho por comprender acerca de los patrones de diversidad florística y la estructura de muchos parches disyuntos de los bosques estacionalmente secos del sistema del Marañón. El propósito de este trabajo es contribuir al conocimiento de la diversidad y niveles de endemismo de los Bosques de Jaén. Adicionalmente, dada la disponibilidad de información generada se muestran algunos parámetros preliminares vinculados a la estructura, cuyos resultados propicien las bases para su conservación.

\section{Materiales y métodos} Descripción de la zona de estudio
La provincia de Jaén es una de las 13 provincias que conforman la región Cajamarca; se encuentra ubicada al Norte de esta región y posee una superficie de $5232 \mathrm{~km}^{2}$. Por el Norte limita con la provincia de San Ignacio, al Sur con la provincia de Cutervo, al Oeste con la provincia de Huancabamba de la región Piura y por el Este con las provincias de Bagua y Utcubamba de la región Amazonas. Esta provincia está conformada por doce distritos. Su capital, la ciudad de Jaén se encuentra a $295 \mathrm{~km}$ de la ciudad de Chiclayo y a $1060 \mathrm{~km}$ de la ciudad de Lima, entre las coordenadas $05^{\circ} 42^{\prime}$ de Latitud Sur y $78^{\circ} 48^{\prime}$ de Longitud Oeste. Posee una superficie de $537.25 \mathrm{~km}^{2}$, y representan el $10.27 \%$ de la superficie de la provincia (Anónimo, 2004).

La zona de estudio se encuentra ubicada en los sectores El Huito, San Isidro, Shanango, y Mochenta,

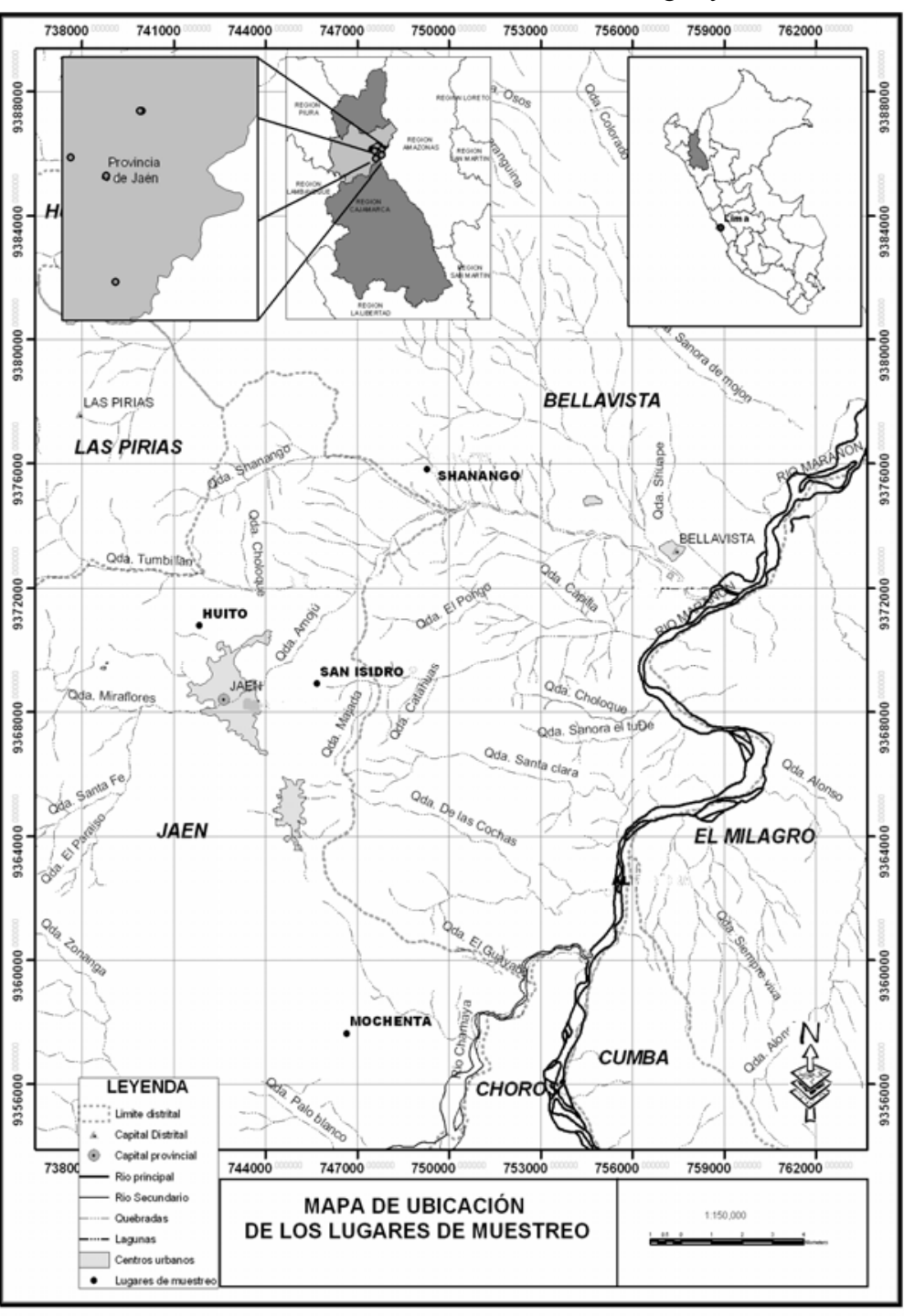

Figura 1. Mapa de ubicación de los cuatro sitios muestreados (Shanango, El Huito, San Isidro y Mochenta). 
del distrito y provincia de Jaén, Región Cajamarca. Se halla entre los $5^{\circ} 38^{\prime}$ y $5^{\circ} 48^{\prime}$ de latitud Sur y $\operatorname{los} 78^{\circ}$ $41^{\prime}$ y $78^{\circ} 48^{\prime}$ de longitud oeste (Figura 1). Las alturas oscilan entre 615 y $1080 \mathrm{~m}$.

La zona de estudio se encuentra dentro de la zona de vida Bosque muy seco tropical (bms- T); está ocupada por especies perennifolias y caducifolias de porte arbóreo y arbustivo. El clima es seco con una temperatura media anual que oscila entre 24 a $26^{\circ} \mathrm{C}$, con máximas que alcanzan los $30{ }^{\circ} \mathrm{C}$ y mínimas que están alrededor de 20 a $21{ }^{\circ} \mathrm{C}$; la temperatura promedio se mantiene a lo largo del año. La precipitación promedio anual varía desde $350 \mathrm{~mm}$ hasta $1000 \mathrm{~mm}$ y presenta un periodo seco de menores precipitaciones entre los meses de mayo a octubre y de mayores precipitaciones entre octubre y abril (Anónimo, 2004).

El escenario fisiogeográfico presenta rasgos geomorfológicos que son el resultado de la evolución originada por factores tectónicos y erosionales. Está conformada por laderas moderadamente empinadas de $25-50 \%$ de pendiente y laderas muy empinadas mayores de $70 \%$. La zona de estudio ocupa el ámbito de la cuenca Amojú que se encuentra disectada por el cauce del río del mismo nombre, fuente primaria proveedora de agua para riego y para satisfacer las necesidades de las poblaciones humanas asentadas en el valle de Jaén. El recorrido de las aguas del río Amojú son de Norte a Sur y aguas abajo confluyen en la margen izquierda del río Marañón que es el colector principal.

El valle de Jaén es eminentemente agrícola y en gran parte de su área se ha instalado cultivos de "arroz" Oriza sativa L., que es el más importante. Adicionalmente se cultiva "cacao" Theobroma cacao L. y frutales en menores áreas. Los cultivos de "maíz" Zea maiz Vell., "plátano" Musa spp. y "yuca" Manihot esculenta Cif., son principalmente de consumo familiar y/o para el mercado local. Las zonas de ladera presentan agricultura restringida dedicada especialmente al cultivo de "maíz" y a la siembra de pasto para sostener ganadería extensiva.

Todos los relictos evaluados están siendo utilizados actualmente como áreas de pastoreo de ganado vacuno. Por ejemplo, alrededor del relicto ubicado al Noroeste de la ciudad de Jaén, en el sector El Huito se han establecido pastos que regularmente son quemados y presentan una formación tipo sabana con especies arbóreas de Cybistax antisyphilitica (C. Martius) C. Martius ex A. DC., Luehea paniculata Mart. \& Zucc., Aspidosperma polyneuron Müll. Arg. "acerillo", Acacia macracantha H. \& B. ex Willd. "faique o huarango", Cordia iguaguana Melch. ex I.M. Johnst. "iguaguana", Hura crepitans L. "catagua", Sapindus saponaria L. "choloque", Capparis scabrida Kunth "zapote o zapotillo", Pseudobombax sp. entre las más conspicuas y especies arbustivas de Boungainvillea peruviana
Humb. \& Bonpl., Malphygia glabra L., Cyathostegia matthewsii (Benth.) Schery, Tournefortia hirsutissima L. (Marcelo-Peña, 2007)

\section{Metodología}

La investigación se inició en los BTES de Jaén, con el desarrollo de inventarios botánicos rápidos (IBR) a partir de Abril y Julio del 2006 en los sectores El Huito, San Isidro1, Yanuyacu y Shanango, culminando las evaluaciones en Diciembre del mismo año. En Enero y Abril del 2007 se evaluaron los sectores: El Almendral, Mochenta, Linderos Alto y San Isidro2. Procesada la información de los IBR, se escogieron los sectores que presentaron mayor diversidad y mayores niveles de endemismos. Dado que en el ámbito la vegetación leñosa está confinada en parches remanentes por la alteración antropogénica y que estos son escasos y de pequeña extensión, los transectos fueron emplazados en aquellos que tenían una extensión suficiente.

En cada sector se procedió con la metodología propuesta por Gentry (1982) y discutida por Phillips \& Miller (2002a), que consiste en el levantamiento de la vegetación en unidades de 0.1 ha, conformadas por 10 transectos de 2 × $50 \mathrm{~m}$. Para el levantamiento se registraron todas las especies con diámetros a la altura del pecho $(1.37 \mathrm{~m}) \geq 2.5 \mathrm{~cm}$, se incluyó árboles, arbustos, lianas y arbustos escandentes. Simultáneamente se colectó el material botánico de cada uno de los individuos hallados. Los ejemplares fueron procesados y montados siguiendo la metodología descrita por Rodríguez \& Rojas (2002). La identificación del material botánico se realizó en el Herbario MOL y USM mediante la utilización de claves taxonómicas, comparación con especimenes existentes y revisión de material bibliográfico. Las colecciones botánicas se encuentran depositadas en el Herbario MOL.

Para la determinación de las especies endémicas y sus datos de distribución se tomo como referencia las colecciones de los herbarios USM y MOL (acrónimos según Holmgren et al., 1990), literatura especializada (León et al., 2006; Brako \& Zarucchi, 1993; Valencia et al., 2000), así como monografías recientes (Pendry, 2003; Barneby \& Grimes, 1997) y de bases de datos en línea como W3TROPICOS (http://mobot.mobot.org/W3T/Search/vast.html).

\section{Análisis de los datos}

Para cada sector evaluado se determinaron tres variables, siguiendo los criterios de Phillips \& Miller (2002a) y de Antón \& Reynel (2004):

Variables vinculadas a la diversidad: número de individuos por ha, número de especies por muestra, número de familias por muestras y curva especies áreas. Se hallaron también los valores de los índices de diversidad de Fisher y el índice de diversidad de Shannon \& Wiener. 
Variables vinculadas a la composición florística: familias, géneros y especies más abundantes, especies endémicas y raras, riqueza por hábitos de crecimiento. Variables estructurales: de modo preliminar y complementario se hallaron algunos parámetros estructurales básicos. Los datos de la estructura fueron introducidos en una base da datos y se calculó abundancia, área basal, frecuencia, distribución diamétrica y el índice de valor de importancia IVI (Matteucci \& Colma, 1982). Los 40 transectos estudiados fueron analizados con el Software PRIMER v.5 (Clarke \& Gorley, 2001), para obtener las curvas de acumulación especie-área y los índices de diversidad de Fisher y Shanon-Wiener.

\section{Resultados}

\section{Diversidad y composición florística}

En los cuatro sectores muestreados $(0.4$ ha en total) se registraron un total de 52 especies, con un valor promedio de 28. El Huito es el sector con mayor riqueza de especies (30), le siguen en orden decreciente San Isidro (29), Mochenta (28) y Shanango (25). Según las formas de vida 29 especies son árboles, 17 especies son arbustos, cuatro especies escandentes y dos especies de lianas.

En total se registraron 27 familias. En el Huito se encontró 21 familias, 16 en San Isidro, 17 en Mochenta y 15 familias en Shanango. La familia más diversa es Leguminosae con 13 especies, le siguen en orden decreciente Cactaceae con ocho y Euphorbiaceae con tres especies, las demás familias presentan dos y una especie (Figura 2). Son familias raras del bosque estacionalmente seco de Jaén Rhamnaceae, Moraceae, Celastraceae y Verbenaceae. Maytenus octogona (L' Her.) DC., Aloysia scorodonioides (Kunth) Cham., Sageretia elegans (Kunth) Brongn. y Heteropsis sp. son especies raras, sólo se registró un individuo cada uno. Bauhinia aculeata L. y Coursetia maraniona Lavin, también son especies raras de la familia Leguminosae.

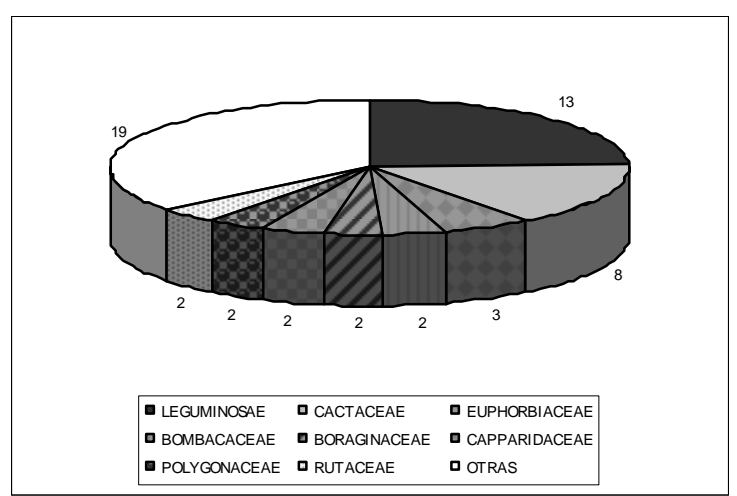

Figura 2. Familias con mayor número de especies de cuatro muestras de 0.1 ha de bosques estacionalmente secos alterados del distrito de Jaén.

\section{Índices de diversidad}

Se tomó como referencia el índice de diversidad de Fisher y el índice de diversidad de Shannon-Wiener. El índice de Fisher caracteriza comunidades bióticas que contienen pocas especies que son abundantes y muchas que son escasas, además, valoriza la diversidad independientemente del área y del tamaño de la muestra. El índice de diversidad de Shanon \& Wiener, mide la heterogeneidad combinando el número de especies y la igualdad o desigualdad de la distribución de los individuos de las diversas especies (Krebs, 1989). El valor de Fisher para El Huito es de 8.5, San Isidro 8.4, Mochenta 8.1 y Shanango 6.2. Los valores del índice de diversidad de Shannon-Wiener son de 4.1 para San Isidro, Mochenta 3.7, Shanango 3.5 y El Huito 2.9.

\section{Curvas de acumulación de especies}

En todos los sectores evaluados la curva de acumulación especie-área (Figura 3), tiene un incremento de las especies hasta alcanzar un área de $800 \mathrm{~m}^{2}$. A medida que se va incrementando el área se nota una tendencia a la saturación de la curva. Esta característica es más evidente en San Isidro y Shanango. No obstante, para El Huito y Mochenta, con el incremento del área, también se incrementa el número de especies.

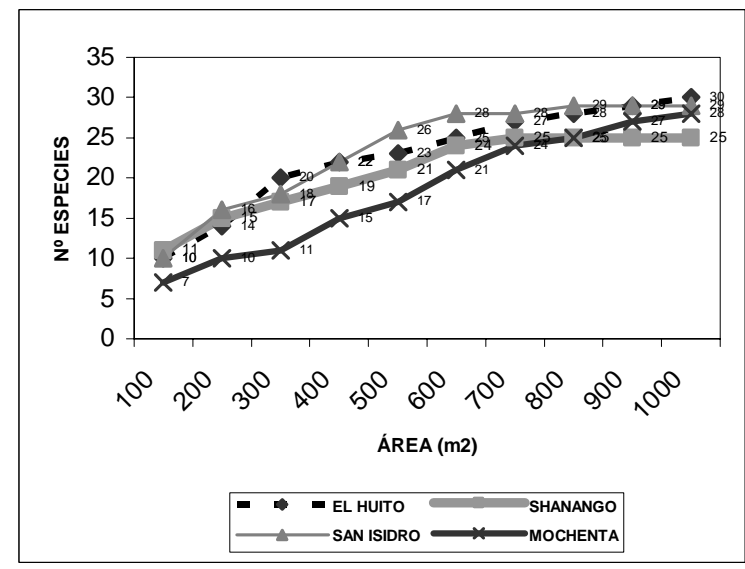

Figura 3. Curva de acumulación especie-área de 10 transectos de $50 \mathrm{~m} \times 2 \mathrm{~m}$, en cuatro sectores de bosques estacionalmente secos alterados del distrito de Jaén.

\section{Endemismo}

Se registró 15 especies endémicas en las 0.4 ha, Shanango y Mochenta son los sectores que presenta los valores más elevados en endemismos con 10 especies en muestras de 0.1 ha. Le siguen San Isidro con ocho especies y El Huito con siete especies (Tabla 1). Praecereus euchlorus subsp. jaenensis (Rauh \& Backeb.) Ostolaza, Jatropha weberbaueri Pax \& K. Hoffm., Tetrasida chachapoyensis (Baker f.) Fryxell $\&$ Fuertes y Ruprechtia aperta Pendry, son especies 
endémicas que se pueden encontrar frecuentemente en los sectores muestreados.

Tabla 1. Especies endémicas registradas en muestras de 0.1 ha en bosques estacionalmente secos alterados de cuatro sectores del distrito de Jaén.

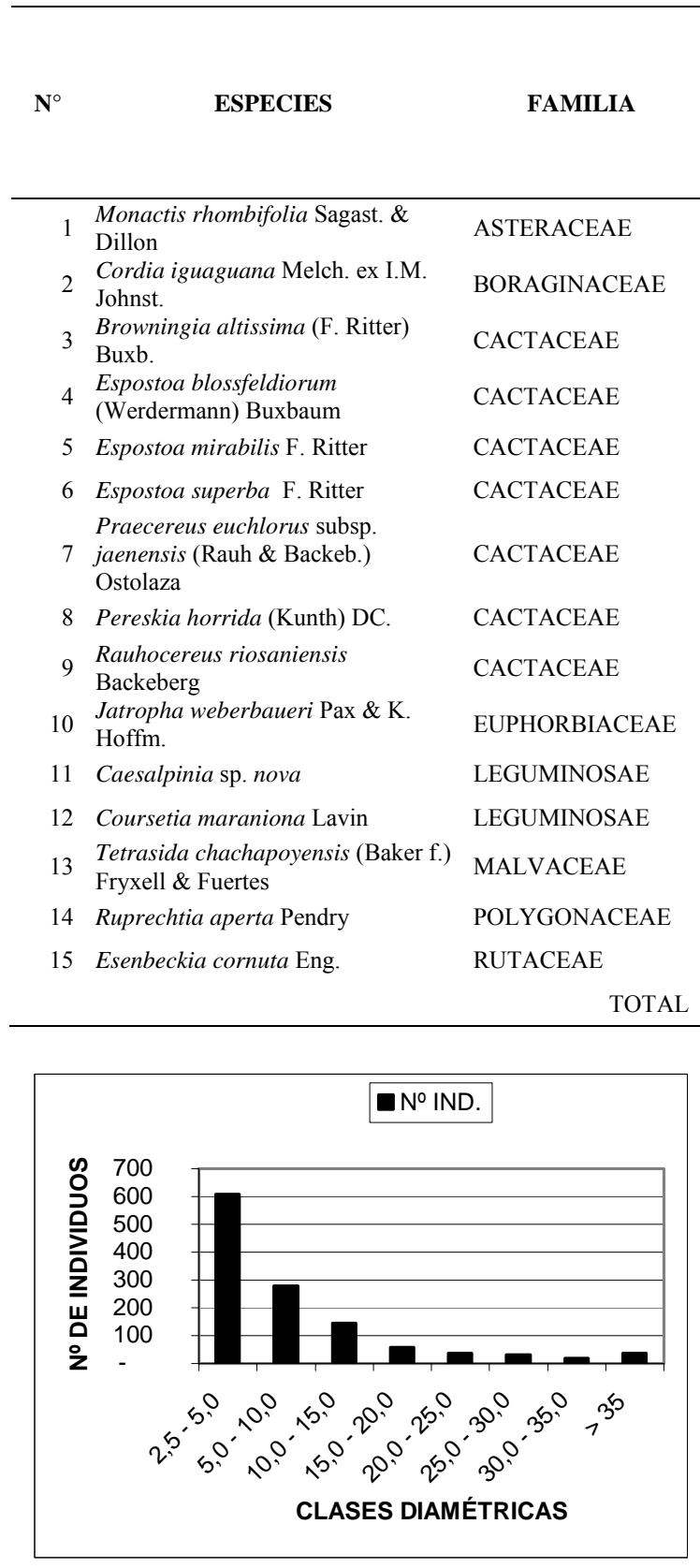

Figura 4. Curva de distribución de individuos por clases diamétricas de bosques estacionalmente secos alterados de cuatro sectores del distrito de Jaén.

\section{Fitosociología}

De los cuatro sectores muestreados, se registró un total de 1215 individuos, siendo el promedio por sector de 303. El mayor número de individuos se registró en Shanango con 397, le siguen en orden decreciente El Huito con 280, Mochenta con 278 y San Isidro con 257. Las especies con mayor densidad en el sector Shanango son Cordia iguaguana Melch. ex I.M. Johnst., Esenbeckia cornuta Eng. y T. chachapoyensis. En el Huito, C. iguaguana, Acacia macracantha Humb. \& Bonpl. ex Willd. y Urera caracasana (Jacq.) Griseb. En Mochenta, Browningia
altissima (F. Ritter) Buxb., Croton thurifer Kunth y Caesalpinia sp. nova. En san Isidro, $T$. chachapoyensis, C. iguaguana y $R$. aperta (Tabla 3 y ANEXO 1, 2, 3 y 4).

Según las clases diamétricas, se muestra la tipica "J" invertida (Figura 4), siendo la categoría $\geq 2.5 \mathrm{~cm} \mathrm{y}<5$ $\mathrm{cm}$, en donde se concentra el mayor número de individuos, haciendo un total de $609(50.1 \%)$. En la clase diamétrica $>35 \mathrm{~cm}$ hay 37 individuos (3.04\%).

El área basal de la clase diamétrica $\geq 2.5 \mathrm{~cm}-<5 \mathrm{~cm} \mathrm{de}$ DAP, suman un total de $0.58 \mathrm{~m}^{2}$ $\left(5.89 \mathrm{~m}^{2} / \mathrm{ha}\right)$, en las clases mayores a cinco $\mathrm{cm}$ de DAP se incrementa el área basal, no obstante, el número de individuos disminuye significativamente a medida que se incrementa el diámetro, pero se incremente notablemente el área basal (Figura 5). La clase diamétrica $>35 \mathrm{~cm}$ de DAP, con 37 individuos suman un área basal de 10.11 (101.17 $\left.\mathrm{m}^{2} / \mathrm{ha}\right)$.

El área basal total de los 1215 individuos es de $19.81 \mathrm{~m}^{2}, \quad\left(49.54 \mathrm{~m}^{2} / \mathrm{ha}\right)$ y el promedio por sector es de $4.95 \mathrm{~m}^{2}$. El valor más elevado de área basal fue para el sector de Mochenta con $11.92 \mathrm{~m}^{2}$ (119.2 $\left.\mathrm{m}^{2} / \mathrm{ha}\right)$. Sigue El Huito con $4.3 \mathrm{~m}^{2}\left(43.18 \mathrm{~m}^{2} / \mathrm{ha}\right)$. San sidro con $2.1 \mathrm{~m}^{2}\left(21.4 \mathrm{~m}^{2} / \mathrm{ha}\right)$ y Shanango con $1.4 \mathrm{~m}^{2}$ $\left(14.4 \mathrm{~m}^{2} / \mathrm{ha}\right)$. Las especies con mayor área basal por sector son: C. iguaguana, Anadenantera colubrina (Vell.) Brenan y Aspidosperma polyneuron Müll. Arg., en el sector El Huito. En Shanango, C. iguaguana, Ceiba insignis (Kunth) P.E. Gibbs \& Semir y E. cornuta. En San Isidro C. iguaguana, Priogymnanthus apertus (B. Ståhl) P.S. Green y Capparis flexuosa (L.) L.y en el sector Mochenta $B$. altissima, $C$. insignis y A. colubrina. Las especies que poseen los valores más altos de área basal son, $B$. altissima con $4.5 \mathrm{~m}^{2}$. C. insignis con $4.2 \mathrm{~m}^{2}, C$. iguaguana con $3.3 \mathrm{~m}^{2}$., A. colubrina con $1.6 \mathrm{~m}^{2}$, Sideroxylon obtusifolium (Roem. \& Schult.) T.D. 
Penn., con $0.6 \mathrm{~m}^{2}$ y P. apertus con $0.57 \mathrm{~m}^{2}$. (Tabla 2 y ANEXO 1, 2, 3 y 4).

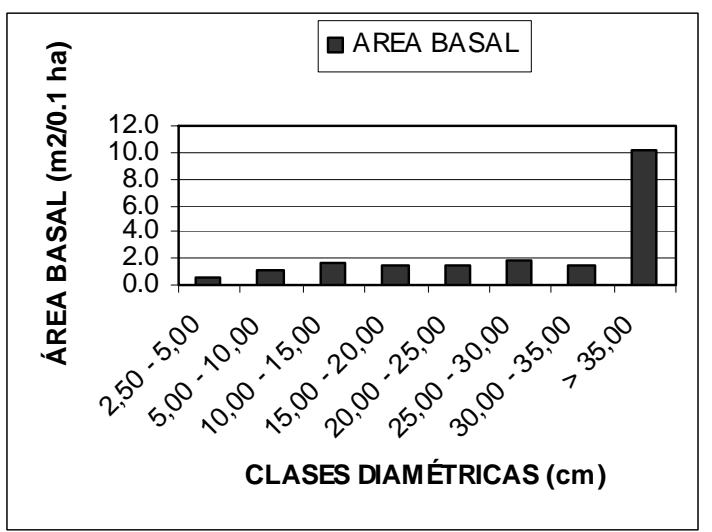

Figura 5. Curva de distribución de área basal $\left(\mathrm{m}^{2}\right)$ por clases diamétricas de bosques estacionalmente secos alterados de cuatro sectores del distrito de Jaén.

Las especies con mayor índice de valor de importancia (IVI) considerando cada uno de los sectores evaluados son, C. iguaguana, $A$. macracantha y A. colubrina para el sector El Huito. C. iguaguana, E. cornuta y T. chachapoyensis para Shanango. T. chachapoyensis, $C$. iguaguana y $P$. apertus para San Isidro; B. altissima, C. insignis y A. colubrina para Mochenta (Tabla 2 y ANEXO 1, 2, 3 y 4).

\section{Estratificación vertical}

La estructura vertical está conformada por dos estratos principales. El primer estrato corresponde al dosel arbustivo que alcanza alturas de dos hasta cuatro metros, las especies que dominan este estrato son: $C$. thurifer, R. aperta, J. weberbaueri, Acacia weberbaueri Harms, U. caracasa, Erythroxyllum sp, $P$. euchlorus subsp. jaenensis, Rahuoceresu riosaniensis Backeberg, Cyatosthegia mattewsii (Benth.) Schery, Caesalpinia sp. nova, M. octogona. El segundo estrato está conformado por especies que alcanzan alturas entre $6-12 \mathrm{~m}$ de alto siendo las más conspicuas C. flexuosa, Zanthoxyllum rigidum Humb. \& Bonpl. ex Willd., Cordia saccellia Gottschling \& J.S. Mill., B. altissima, A. macracantha, $C$. iguaguana, C. insignis, Eriotheca discolor (Kunth) A. Robyns, E. cornuta, P. apertus, Celtis iguanea (Jacq.) sarg., A. polyneuron y A. colubrina.

\section{Discusión \\ Diversidad alfa y composición florística}

En general los bosques tropicales estacionalmente secos (BTES) presentan la mitad o un tercio del total de especies de plantas leñosas en comparación con los bosques húmedos y muy húmedos (Gentry, 1995). En el Neotrópico el número de especies en muestreos de 0.1 ha, registrando todos los individuos con DAP $\geq$ $2.5 \mathrm{~cm}$, fluctúa entre 21 y 121 con un valor promedio de 67 especies. Haciendo un contraste entre los bosques en estudio y los bosques estacionalmente secos de Centro y Sudamérica (Tabla 3), los BTES de Jaén estudiados presentan menor diversidad y densidad, por estar sometidos a impactos frecuentes generados por la presencia de pastoreo de ganado vacuno y alta demanda de leña por pobladores locales.

En el Perú en los Cerros de Amotape, región Tumbes, Gentry (1995) reporta un total de 401 individuos de 57 especies y en la región San Martín, provincia de Tarapoto, reportó un total de 520 individuos y 102 especies. Ambas zonas son mayores en densidad y riqueza de especies que los valores obtenidos en el presente estudio. Tarapoto presentan una composición florística de naturaleza transicional con frecuencia de familias mayormente amazónicas (Sapotaceae, Meliaceae, Celastraceae, Arecaceae, Rubiaceae, Anacardiaceae) y es difícil relacionarla con formaciones de BTES en la región. LinaresPalomino et al. (2004), y Bridgewater et al. (2003), reportaron que hay una similitud muy baja de los bosques de esta región con otros BTES en la costa de Ecuador y Perú y de los Andes peruanos.

Leguminosae y Bignoniaceae son dos familias de plantas que tienden a dominar los BTES de sudamérica y en éstas se encuentran representadas la mayoría de las especies (Gentry, 1995). Sin embargo, ninguna de estas familias dominó en los sectores evaluados. Boraginaceae, Malvaceae y Cactaceae son las familias con mayor valor de importancia para los bosques de Jaén. Leguminosae es la más diversa y solo en los sectores Mochenta y El Huito dominó después de Boraginaceae y Cactaceae. Bignoniaceae es una familia que presenta una sola especie con escasos individuos (en Mochenta y Shanango esta especie es más conspicua), debido a que los bosques en estudio son secundarios, Gentry buscó áreas no intervenidas donde la presencia de esta familia es más conspicua.

En el bosque de Mochenta (15 ha aprox.) dominó Cactaceae, esta comunidad vegetal se ha desarrollado en piedemonte ligeramente inclinado, con suelos franco arenosos con presencia de piedras y gravas subangulares, características que no comparte con los otros sectores, en donde el dominio ha sido de la familia Boraginaceae.

Gentry (1995), indica que los géneros más comunes en los bosques secos del Neotrópico son Tabebuia, Casearia, Bauhinia, Trichilia, Erythroxyllum, Randia, Hippocratea, Serjania, Croton y Zanthoxylum; estos patrones de diversidad son semejantes con los resultados obtenidos excepto por Casearia, Trichilia, Randia y Hippocratea que no han sido registrados en los sectores evaluados. Los géneros más comunes en los bosques de Jaén son Cordia, Tetrasida, Browningia, Croton, Priogymnathus, Praecereus y Ruprechtia. 
Gentry (1995) indica que las familias de lianas más dominantes en los bosques estacionalmente secos del Neotrópico son Bignoniaceae y Sapindaceae, mientras que las más diversas son Bignoniaceae y Malphygiaceae. En los bosques de Jaén las lianas son raras, en este estudio solo se registró un individuo de Serjania grammatophora Radlk. (Sapindaceae) en El Huito y un solo individuo de Heteropsis sp. (Malpighiaceae) en Shanango, la escases de lianas de los BTES de Jaén se debe a su naturaleza predominantemente secundaria.

\section{Índices de diversidad}

Existen escasas referencias sobre los índices de diversidad en general para los BTES del Perú. Gentry (1995), enfatiza los valores de diversidad alfa (número de especies), la composición florística y la estructura del Bosques, al igual que Bridgewater et al (2003); sin embargo, no citan los índices de diversidad. LealPinedo \& Linares-Palomino (2005), en los bosques secos de la Reserva de Biosfera del Noroeste (Piura y Tumbes), en 16 parcelas de una hectárea, evaluando todos las especies $\geq 10 \mathrm{~cm}$ de DAP, obtuvo valores de diversidad de Shannon-Wiener que oscilan entre 1.17 y 3.03 y valores del índice de diversidad de Fisher que oscilan entre 1.41 y 9.96 .

El análisis de los índices de diversidad muestra marcados contrastes. El índice de diversidad de Fisher indica que El Huito (8.5) es el más diverso y Shanango (6.2) el menos diverso. Sin embargo, con el índice de diversidad de Shanon-Wiener, El Huito (2.9) es diverso, pero con mucha heterogeneidad de abundancia de especies. Marcelo-Peña (2007), a través de inventarios botánicos concluye que El Huito y San Isidro (47 y 43 especies para el primero y segundo), son los sectores más diversos, mientras que Shanango con 36 especies es el menos diverso. Note que el índice de diversidad de Fisher permite hacer comparaciones relativamente fieles del nivel de diversidad entre sitios que con una misma área pueden variar en términos de abundancia, a diferencia de los otros índices (Shannon-Wiener, Simpson, Margalef) que también se utilizan para cuantificar la diversidad.

Según Knight (1975, citado por Rabelo et al. 2002) el índice de Shanon-Wiener para los bosques tropicales oscila entre 3.83 y 5.85 . Considerando las limitaciones ambientales y el grado de perturbación que sufren los bosques estacionalmente secos de Jaén, estos presenta elevada diversidad.

\section{Curvas de acumulación especies-área}

En la curva especie-área, la incorporación de nuevas especies se relaciona con el área de muestreo. Al principio, se registró especies comunes y la adición de especies al inventario se produce rápidamente; por tanto, la pendiente de la curva comienza siendo elevada para los cuatro sectores. A medida que prosigue el muestreo son las especies raras, las que hacen incrementar levemente la curva sin llegar a estabilizarse. En los sectores de El Huito y Mochenta, se observa que la curva especie-área sigue una trayectoria ascendente, por tanto aún estamos lejos de alcanzar el punto en que se pueda estimar con mayor precisión la riqueza de especies para estos sectores; Marcelo-Peña (2007), apoya esta afirmación, quien a través de inventarios botánicos rápidos, registró 47 especies para el primero y 50 especies para el segundo. En Shanango la curva se estabiliza a partir de $\operatorname{los} 800 \mathrm{~m}^{2}$.

Las diferencias en la diversidad de especies y en el número de especies compartidas entre los sectores estudiados sugieren una distribución heterogénea florísticamente acentuada en los sectores de San Isidro, El Huito y Mochenta, por encontrar altos valores en las clases de frecuencias I y II (Matteucci \& Colma, 1982). En estos sectores más del 50\% del total de las especies encontradas poseen muy pocos individuos (menos de cinco), cuya distribución espacial aumenta la diversidad; no obstante, que en Shanango la distribución es más homogénea. Probablemente, la alteración antropogénica, así como, factores micro-ambientales en pequeña escala, tales como diferencias en la composición del suelo, en la topografía del terreno o de altitud entre los sitios de muestreo (Lieberman et al., 1985) podrían estar determinando la heterogeneidad observada en la distribución de las especies.

\section{Endemismos}

C. iguguana, T. Chachapoyensis y B. altissima son endémicas y presentaron mayor peso ecológico. $C$. iguaguana es la más importante es el Huito y Shanango, en San Isidro está detrás de $T$. chachapoyensis, C. iguaguana es una especie restringida a las regiones de Cajamarca, Amazonas y San Martín. T. chachapoyensis es la más importante en San Isidro y está restringida a las regiones de Cajamarca y Amazonas, mientras que B. altiissima es las más importante en Mochenta y se encuentra restringida solo a los BTES del distrito de Jaén.

Arakaki et al. (2006) no le otorgaron categoría de amenaza a E. blossfeldiorum, E. mirabilis, E. superba, Pereskia horrida y $R$. riosaniensis. E. bolssfeldiorum se encuentra distribuida en Cajamarca y Piura, en la zona de estudio se la observó en Mochenta y Shanango pero con muy pocos individuos y la consideramos en peligro (EN). E. mirabilis se encuentra distribuida en Amazonas y Cajamarca, en la zona de estudio sólo se la registró en Mochenta con un solo individuo y coincidimos con Ostolaza (1997) que la categorizó en peligro (EN). E. superba se encuentra restringida a los bosques estacionalmente secos de Jaén y crece muy dispersa, en los inventarios solo se registró un individuo en Mochenta, sugerimos que se encuentra en peligro crítico (CR). Concordamos con Ostolaza (1997) que también la consideró en peligro (EN) a Rahuocereus riosaniensis; en nuestra investigación la especie fue registrada en los sectores de El Huito, San Isidro y 
Shanango, con una población aproximada de 80 individuos/ha.

Como registro interesante de los sectores estudiados destacamos el hallazgo de Esenbeckia cornuta, que no ha sido reportada en 156 años, desde la colección tipo realizada por Warscewicz en 1851. León et al. (2006), no le otorgaron a E. cornuata una categoría de amenaza por presentar datos insuficientes. Sin embargo, sugerimos que se encuentra en peligro crítico de extinción (CR), por su rango geográfico restringido solo al sector de Shanango (con un área de ocupación menor a $10 \mathrm{~km}^{2}$ ), por la severa fragmentación de su hábitat y la eminente desaparición de sus poblaciones por agricultura y ganadería. La densidad de esta especie por clase diamétrica para individuos $\geq 2.5$ pero menores de cinco centímetros de DAP es de 570/ha, para individuos $\geq 5$ pero menor de $10 \mathrm{~cm}$ de DAP es de 170/ha y 20 individuos/ha para los registros sobre $10 \mathrm{~cm}$ de DAP.

Aguirre \& Kvist (2005), en los bosques secos del Sur Occidente del Ecuador que incluye las provincias de Loja, El Oro, Guayas, Manabí, Esmeralda y Los Ríos, registraron 15 especies endémicas. En contraste los bosques del distrito de Jaén presentan valores sorprendentemente altos en especies endémicas, en apenas 0.4 hectáreas hemos registrado el mismo número.

Marcelo-Peña (2007) a través de inventarios botánicos rápidos registró 30 especies endémicas en ocho sectores, en El Huito registró ocho especies endémicas, 12 en San Isidro, 14 en Shanango y Mochenta. En el presente estudio se confirma que los sectores de Mochenta y Shanango pueden tener los bosques con mayores endemismos en Jaén y que los bosques estacionalmente secos de este distrito tienen prioridad de conservación por presentar los valores más altos en endemismos para los BTES del Perú y Sur del Ecuador.

\section{Fitosociología}

La densidad de especies en los BTES de Centro y Sudamérica oscila entre 141 y 597 (Gentry 1995), siendo los bosques de México, Colombia y Costa Rica los que superan en densidad a los bosques estudiados. En contraste con Ecuador, Argentina y Paraguay cuya densidad es menor. Las especies con mayor abundancia son C. iguaguana con 261 individuos y representa el $21.4 \%$ del total de individuos, le sigue $T$. chachapoyensis con 105 individuos y representa el $8.6 \%$, E. cornuta con 77 individuos que representan $6.3 \%$. C. thurifer y B. altissima con 75 individuos cada una y representan el $6.1 \%$. La preferencia de estas especies por un tipo especial de suelo aún no ha sido documentada y puede influenciar en la heterogeneidad espacial de los bosques en estudio.

La densidad en los sectores evaluados es marcadamente diferente; en Shanango la mayor densidad fue aportada por seis especies que presentan más de 20 individuos y 10 especies presentan menos de cinco; esta característica no se presenta en El Huito donde sólo dos especies presentan densidades mayores a 20 individuos y 23 especies presentan menos de cinco. En San Isidro solo tres especies presentan más de 20 individuos y 14 especies menos de cinco. En Mochenta, también seis especies aportan más de 20 individuos pero 18 especies con menos de 5 individuos. Se nota que existe una mayor presencia de especies con pocos individuos que dan valores pequeños en IVI mientras que entre 2 y 6 especies poseen altas densidades y poseen los valores más altos en IVI (ANEXO 1, 2, 3 y 4).

En tres de los sectores evaluados C. iguaguana, presenta un peso ecológico alto y es la más conspicua. $U$. caracasana es un arbusto frecuente solo en El Huito, en los demás sectores no fue registrada. El mismo caso se da para E. cornuta, especie arbórea que sólo se registró en Shanango. E. cornuta es una especie con una población homogénea de aproximadamente 760 individuos/ha, no obstante, que por presentar distribución restringida tan solo al sector evaluado, su conservación merece especial atención.

El valor de área basal hallado está lejos del promedio $(30 \mathrm{~m} 2 / \mathrm{ha})$ encontrado con otros estudios (Gentry, 1995). El encontrar numerosos individuos delgados y pocos individuos gruesos, provocó la concentración del área basal en las clases diamétricas inferiores (menores de cinco $\mathrm{cm}$ de DAP), obteniéndose valores de área basal bajos.

Considerando el área basal total se notan diferencias, los sectores con menor área basal fueron Shanango $\left(1.4 \mathrm{~m}^{2}\right)$ y San Isidro $\left(2.1 \mathrm{~m}^{2}\right)$, evidenciando que estos fueron los más degradados. El área basal total en Mochenta fue de $11 \mathrm{~m}^{2}$, este valor se ha logrado por la abundancia de la cactácea arbórea $B$. altissima $\left(4.1 \mathrm{~m}^{2}\right)$ que no se utilizada con fines combustibles ni maderables, que son las principales causas de la reducción del área basal y pérdida de la diversidad.

Actualmente son muy escasos los individuos de especies de importancia económica con diámetros mayores a $40 \mathrm{~cm}$ de DAP; hace ya más de cuatro décadas que Tabebuia chrysantha (Jacq.) G. Nicholson "Guayacán", C. iguaguana "iguaguana" $y$ A. polyneuron "acerillo" fueron taladas para la construcción de viviendas (utilizados en la elaboración de columnas, vigas, tijerales y dinteles) y en la última década para la industria del parquet. Sus poblaciones se han reducido a individuos jóvenes que no alcanzan alturas mayores de $20 \mathrm{~cm}$ de DAP para $T$. chrysantha y de $40 \mathrm{~cm}$ DAP para A. polyneuron. $C$. iguaguana hasta la fecha se sigue aprovechando para uso combustible (leña para hornos de pan, hornos de ladrillo y cocción de alimentos), sus poblaciones han podido perdurar en su hábitat por la buena capacidad de rebrote (entre tres y 10 rebrotes que no superan los $10 \mathrm{~cm}$ de diámetro). 


\section{Conclusiones}

De acuerdo a los levantamientos efectuados, los BTES del distrito de Jaén presentan menor diversidad, densidad y área basal que otras muestras BTES de países vecinos, estos bosques están sometidos al pastoreo de ganado vacuno y por la alta demanda de leña por pobladores locales. Cordia iguaguana, Tetrasida chachapoyensis y Browningia altissima son endémicas restringidas a los BTES del Marañón y son las que alcanzaron los mayores valores en IVI. Boraginaceae, Malvaceae y Cactaceae son las familias con mayor valor de importancia. Los resultados confirman que la zona presenta valores sorprendentemente altos en endemismo en contraste con los BTES interandinos y los BTES orientales de nuestro país y los BTES del sur occidente del Ecuador. Por lo tanto, su conservación merece especial consideración.

\section{Agradecimientos}

A Carlos Ostolaza por la determinación de las especies de la familia Cactaceae. A James Rivera por la elaboración del mapa de la zona de estudio; Duber Elera de la Universidad Nacional de Cajamarca, Ulli Hipler y T. Wendland de la Universidad de Göttingen por su colaboración en el trabajo de campo.

\section{Literatura citada}

Aguirre M. A. \& Kvist L. P. 2005. Composición florística y estado de conservación de los bosques secos del suroccidente del Ecuador. Lyonia Vol.8 (2)

Anónimo. 2004. Atlas regional del Perú. Tomo 13: Cajamarca. Imagen geográfica, estadística, histórica y cultural. Ediciones PEISA 2003. Perú.

Antón D. \& Reynel C. 2004. Relictos de bosque de excepcional diversidad en los Andes Centrales del Perú. Universidad Nacional Agraria La Molina. Herbario de la Facultad de Ciencias Forestales.

Arakaki M., Ostolaza C., Cáceres F.\& Roque J. 2006. Cactaceae endémicas del Perú. Rev. peru biol., vol.13, no.2, p.193-219.

Barneby R.C. \& Grimes J.W. 1997. Silk Tree, Guanacaste, Monkey's Earring. A generic system fro the synandrous Mimosaceae of the Americas. Part II: Pithecellobium, Cojoba \& Zygia. Mem. New York Bot. Gard. 74 (2).

Berry P.E. 2002. Diversidad y endemismo en los bosques neotropicales de bajura. Chapter 4, pp. 83-96. In: Guariguata M.R. \& Catan G.H. (Eds.), Ecología de Bosques Neotropicales. Libro Universitario Regional, Cartago, Costa Rica.

Brako L. \& Zarucchi J.L. 1993. Catalogue of the flowering plants and Gymnosperms of Peru. Monogr. Syst. Bot. Missouri Bot. Gard.

Bridgewater S., Pennington R.T., Reynel C.A., Daza A. \& Pennington T.D. 2003. A preliminary floristic and phytogeographic analysis of the woody flora of seasonally dry forest in northern Peru. Candollea. 58: 129-148.

Clarke K.R. \& Gorley R.N. 2001. PRIMER v5. User Manual/Tutorial. Primer-E Ltd. Plymouth, UK.
Gentry A. H. 1982. Patterns of neotropical plant species diversity. Evolutionary Biology 15: 1-84.

Gentry A.H. 1995. Diversity and floristic composition of Neotropical dry forests. In: Bullock S.H.; H. A. Money H.A. \& E. Medina E.(Eds.) Seasonally Dry Tropical Forests, pp. 146-194. Cambridge, Cambridge University Press.

Holmgren P.K., Holmgren N.H. \& Barnett L.C. 1990. Index herbariorum. Part I: The herbaria of the world. 8th edition. New York Botanical Garden. 693 pp.

Hensold N. 1999. Las Angiospermas endémicas del Departamento de Cajamarca, Perú. Arnaldoa. 6(2): 141184.

INRENA (Instituto Nacional de Recursos Naturales). 1995. Mapa Forestal del Perú escala 1:1000 000 con guía explicativa. Lima, Perú.

Knight D.H. 1975. A phytosociological analysis of epeciesrich tropical forest on Barro Colorado Island, Panamá. Ecol. Monogr. (45): 259-84.

Krebs J. 1989. Ecologhy methodology. Harper \& Row, Publisher, New York, pp 125-166.

León B., Roque J., Ulloa C., Pitman N., Jorgensen M. \& Cano A. 2006. El libro Rojo de las Plantas

Endémicas del Perú. Rev. Per. Biol. Número Especial 13(2): 1-967.

Leal-Pinedo J.M. \& Linares-Palomino R. 2005. Los bosques secos de la Reserva de Biósfera del Noroeste (Perú): Diversidad arbórea y estado de conservación. Caldasia. 27(2):195-211.

Lieberman D., Hartshorn G.S., Lieberman M. \& Peralta R. 1990. Forest Dynamics of La Selva Biological Station, 1969-1985. : 509-521. In: A. Gentry A. (Ed.).Four Neotropical Rainforest.Yale University, New Haven, Connecticut.

Linares-Palomino R. 2004. Los Bosques Tropicales Estacionalmente Secos: II. Fitogeografia y Composición Florística. Arnaldoa. 11(1): 103-138.

Linares-Palomino R. 2005. Los Bosques Secos de la Reserva de Biósfera del Noroeste (Perú): Diversidad Arbórea y Estado de Consevación. Caldasia. 27(2): 195211.

Linares-Palomino R. 2006. Phytogeography and Floristics of Seasonally Dry Forests in Peru. En Pennington, R. T., Lewis, G. P. \& Ratter, J. A. (eds.) Neotropical savannas and Seasonally Dry Forests: Plant Diversity, Biogeography and conservation, pp. 257-279. CRC, Boca Raton, FL.

Marcelo-Peña. J.L. 2007. Inventarios botánicos rápidos en vegetación leñosa de bosques estacionalmente secos del distrito de Jaén, Cajamarca, Perú. Revista Peruana de Biología (en prensa).

Mendoza C.H. 1999. Estructura y riqueza florística del bosque seco tropical en la región Caribe y el valle del río Magdalena, Colombia. Caldasia. 21 (1): 70-94.

Matteucci S. \& Colma A. 1982. Metodología para el estudio de la vegetación. Universidad Nacional Experimental Francisco de Miranda. Coro, Estado Falcón. Venezuela.

Miles L., Newton A.C., DeFries R.S., Ravilious C., May I., Blyth S., Kapos V., Gordon J.E. 2006. A global overview of the conservation status of tropical dry forests. J. Biogeog. 33: 491-505

Murphy P.G. Lugo A.E. 1986. Ecology of Tropical Dry Forest Ann. Re. Ecol. Sist.. 17:67-88 
Murphy P. \& Lugo A.E. 1995. Dry forest of Central America and tha Caribbean. In: Bullock S.H., Mooney H.A. \& Medina E. (Eds.). Seasonally dry tropical forests: 9-34. Cambridge University Press.

Ostolaza C. 1997. Cactus del sur de Cajamarca y del valle del río Saña. Quepo 11

Pendry C. A. 2003. Nine new species of Ruprechtia (Polygonaceae) from Central y South America. Edinburgh Journal of Botany 60(1): 19-42

Phillips O. \& Miller J. 2002a. Global patterns of plant diversity: Alwyn H. Gentry's forest transect data set. Monographs in Systematic Botany from the Missouri Botanical Garden. St. Louis, Missouri. U.S.A.

Pennington R.T., Prado D.E., Pendry. C.A. 2000. Neotropical Seasonally dry forests and quaternary vegetation changes. J. Biogeog. 27: 261-273.

Rabelo F.G., Zarin D.J., De A. Oliveira F. \& Da S. Jardim F.C. 2002. Diversidade, composição florística e distribuição diamétrica do povoamento com dap $>5 \mathrm{~cm}$ em região de estuário no Amapá1, Brasil. Rev. Ciênc. Agrár., Belém. (37): 91-112.
Ratter J.A., Askew G.P., Montgomery R. \& Gifford D.R. 1978. Observations on forests of some meso-trophic soils in central Brazil. Revista Brasil . Bot. I: 47-58.

Rodríguez R.E. \& Rojas G.R. 2002. El Herbario. Administración y manejo de colecciones botánicas. Editado por R. Vásquez M. Jardín Botánico de Missouri - PERÚ.

Sagástegui A., Dillon M.O., Sánchez I., Leiva S. \& Lezama P. 1999. Diversidad florística del Norte del Perú. Tomo I. WWF \& Fondo Editorial Universidad Privada Antenor Orrego. Trujillo, Perú.

Valencia R., Pitman N., León-Yañez S. \&. Jorgensen P.M (Eds.). 2000. Libro de las plantas endémicas del Ecuador 2000. Herbario QCA, Pontificai Universidad Católica del Ecuador, Quito.

Weigend M. 2002. Observations on the Biogeography of the Amotape-Huancabamba Zone in Norther Perú. The Botanical Review. 68(1): 38-54.

Weberbauer A. 1945. El Mundo Vegetal de los Andes Peruanos, Estac. Exper. Agric. La Molina, Editorial Lume, Lima.

\section{Tablas y anexos citados en el texto}

Tabla 2. Resumen de las cinco especies que presentan los valores más altos en abundancia, frecuencia, dominancia e índice de valor de importancia (IVI) en muestreos de 0.1 ha en bosques estacionalmente secos alterados de cuatro sectores del distrito de Jaén.

\begin{tabular}{|c|c|c|c|c|c|c|c|c|c|}
\hline SECT. & ESPECIES & FAMILIA & 皇 & $\frac{\text { s. }}{4}$ & 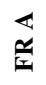 & $\frac{\pi}{2}$ & $\begin{array}{l}\overleftarrow{O} \\
\text { ¿ }\end{array}$ & 品 & $\sum$ \\
\hline \multirow{5}{*}{ 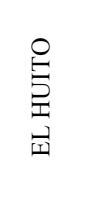 } & Cordia iguaguana & BORAGINACEAE & 152 & 54.29 & 10 & 12.35 & 2.5850 & 59.86 & 126.49 \\
\hline & Acacia macracantha & LEGUMINOSAE & 24 & 8.57 & 8 & 9.88 & 0.1156 & 2.68 & 21.12 \\
\hline & Anadenanthera colubrina & LEGUMINOSAE & 12 & 4.29 & 5 & 6.17 & 0.3262 & 7.55 & 18.01 \\
\hline & Urera caracasana & URTICACEAE & 17 & 6.07 & 7 & 8.64 & 0.0273 & 0.63 & 15.35 \\
\hline & Aspidosperma polyneuron & APOCYNACEAE & 9 & 3.21 & 4 & 4.94 & 0.2395 & 5.55 & 13.70 \\
\hline \multirow{5}{*}{ 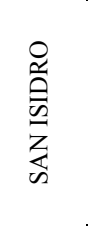 } & $\begin{array}{l}\text { Tetrasida } \\
\text { chachapoyensis }\end{array}$ & MALVACEAE & 52 & 20.23 & 10 & 10.53 & 0.1427 & 6.65 & 37.41 \\
\hline & Cordia iguaguana & BORAGINACEAE & 24 & 9.34 & 4 & 4.21 & 0.3940 & 18.38 & 31.93 \\
\hline & Priogymnanthus apertus & OLEACEAE & 15 & 5.84 & 4 & 4.21 & 0.3893 & 18.15 & 28.21 \\
\hline & Browningia altissima & CACTACEAE & 16 & 6.23 & 8 & 8.42 & 0.2745 & 12.80 & 27.45 \\
\hline & Capparis flexuosa & CAPPARIDACEA & 11 & 4.28 & 5 & 5.26 & 0.3447 & 16.07 & 25.62 \\
\hline \multirow{5}{*}{ 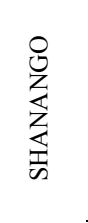 } & Cordia iguaguana & BORAGINACEAE & 85 & 21.41 & 9 & 8.74 & 0.3224 & 22.33 & 52.62 \\
\hline & Esenbeckia cornuta & RUTACEAE & 76 & 19.14 & 7 & 6.80 & 0.1547 & 10.72 & 36.77 \\
\hline & Tetrasida chachapoyensis & MALVACEAE & 49 & 12.34 & 9 & 8.74 & 0.0615 & 4.26 & 25.45 \\
\hline & $\begin{array}{l}\text { Praecereus euchlorus } \\
\text { subsp. jaenensis }\end{array}$ & CACTACEAE & 40 & 10.08 & 8 & 7.77 & 0.0428 & 2.96 & 20.91 \\
\hline & Ceiba insignis & BOMBACACEAE & 3 & 0.75 & 3 & 2.94 & 0.2307 & 15.98 & 19.68 \\
\hline \multirow{5}{*}{ 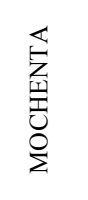 } & Browningia altissima & CACTACEAE & 54 & 19.42 & 9 & 9.78 & 4.1773 & 35.04 & 64.25 \\
\hline & Ceiba insignis & BOMBACACEAE & 2 & 0.72 & 2 & 2.2 & 3.976 & 33.4 & 36.41 \\
\hline & Anadenanthera colubrina & LEGUMINOSAE & 26 & 9.35 & 7 & 7.69 & 1.317 & 11.04 & 28.09 \\
\hline & Croton thurifer & EUPHORBIACEAE & 44 & 15.83 & 9 & 9.78 & 0.2624 & 2.20 & 27.81 \\
\hline & Caesalpinia sp. nova & LEGUMINOSAE & 34 & 12.23 & 8 & 8.70 & 0.3461 & 2.90 & 23.83 \\
\hline
\end{tabular}

$\mathrm{AB} A=$ Abundancia absoluta; $\mathrm{AB} \mathrm{R}=$ Abundancia relativa; $\mathrm{FR} \mathrm{A}=$ Frecuencia absoluta; $\mathrm{FR} \mathrm{R}=$ Frecuencia relativa; $\mathrm{DO} \mathrm{A}=$ Dominancia absoluta; $\mathrm{DO} \mathrm{R}=$ Dominancia relativa 
Tabla 3. Riqueza de familia y especies de los bosques tropicales estacionalmente secos de Centro y Sudamérica en muestreos de 0.1 ha evaluando todos los individuos $\geq 2.5 \mathrm{~cm}$ de DAP. Tomado de Phillips \& Miller (2002a) y Mendoza (1999). Los números en negrita subrayados son los resultados del presente estudio.

\begin{tabular}{|c|c|c|c|}
\hline Localidad & $\mathrm{N}^{\circ}$ Familias & $N^{0}$ Especies & $\mathrm{N}^{\circ}$ Ind. \\
\hline \multicolumn{4}{|l|}{ México (Jalisco) } \\
\hline Chamela (Tierras altas1) & 37 & 91 & 399 \\
\hline Chamela (Tierras altas2) & 34 & 89 & 506 \\
\hline Chamela (Arroyo) & 46 & 103 & 453 \\
\hline \multicolumn{4}{|l|}{ Costa Rica } \\
\hline Guanacaste (Tierrras altas) & 22 & 53 & 437 \\
\hline Guanacaste (Bosque de galerías) & 35 & 63 & 195 \\
\hline \multicolumn{4}{|l|}{ Venezuela } \\
\hline Boca de Uchire, Anzoategui & 20 & 69 & 297 \\
\hline $\begin{array}{l}\text { Estación biol. Los Llanos, } \\
\text { Guarico }\end{array}$ & 21 & 59 & 330 \\
\hline Blohn Ranch, Guarico & 31 & 68 & 306 \\
\hline \multicolumn{4}{|l|}{ Colombia } \\
\hline Coloso, Sucre & 46 & 113 & 339 \\
\hline Galerazamba, Bolivar & 20 & 55 & 396 \\
\hline Forestal Monterrey, Bolívar & 24 & 55 & 471 \\
\hline Finca Bremen, Tolima & 29 & 55 & 597 \\
\hline Finca Cardonal, Tolima & 31 & 60 & 555 \\
\hline \multicolumn{4}{|l|}{ Ecuador } \\
\hline Capeiras, Guayas & 27 & 61 & 304 \\
\hline Perro muerte, Manabí & 33 & 52 & 325 \\
\hline \multicolumn{4}{|l|}{ Perú } \\
\hline Cerros de Amotape, Tumbes & 29 & 57 & 401 \\
\hline Tarapoto, San Martín & 38 & 102 & 520 \\
\hline El Huito, Cajamarca & $\underline{21}$ & $\underline{30}$ & $\underline{280}$ \\
\hline Shanango, Cajamarca & $\underline{15}$ & $\underline{25}$ & $\underline{396}$ \\
\hline San Isidro, Cajamarca & $\underline{26}$ & $\underline{29}$ & $\underline{257}$ \\
\hline Mochenta, Cajamarca & $\underline{15}$ & $\underline{28}$ & $\underline{278}$ \\
\hline \multicolumn{4}{|l|}{ Argentina } \\
\hline Salta Salta & 16 & 25 & 197 \\
\hline Riachuelo, Corrientes & 27 & 47 & 197 \\
\hline \multicolumn{4}{|l|}{ Parque El Rey, Salta } \\
\hline \multicolumn{4}{|l|}{ Bolivia } \\
\hline Chuquimayo, La Paz & 29 & 79 & 465 \\
\hline Santa Cruz, Santa Cruz & 30 & 62 & 170 \\
\hline Quiapaca, Santa Cruz & 27 & 86 & 395 \\
\hline \multicolumn{4}{|l|}{ Paraguay } \\
\hline Fortin Teniente Acosta (900 m) & 11 & 22 & 141 \\
\hline Fortin Teniente Acosta $(600 \mathrm{~m})$ & 9 & 21 & 428 \\
\hline
\end{tabular}

ANEXO 1. Datos fitosociológicos de 10 transectos de $50 \mathrm{~m} \mathrm{x} 2 \mathrm{~m}(0.1$ ha) levantados en el sector El Huito, al noroeste de la ciudad de Jaén.

\begin{tabular}{|c|c|c|c|c|c|c|c|c|c|}
\hline & ESPECIES & FAMILIA & $\begin{array}{c}\text { AB } \\
\mathbf{A}\end{array}$ & AB R & $\begin{array}{c}\text { FR } \\
\text { A }\end{array}$ & $\begin{array}{c}\text { FR } \\
\mathbf{R} \\
\end{array}$ & DO A & $\begin{array}{c}\text { DO } \\
\text { R }\end{array}$ & IVI \\
\hline 1 & Cordia iguaguana Melch. ex I.M. Johnst. & BORAGINACEAE & 152 & 54.29 & 10 & $\begin{array}{c}12.3 \\
5\end{array}$ & $\begin{array}{r}2.5849 \\
5\end{array}$ & $\begin{array}{r}59.8 \\
6\end{array}$ & $\begin{array}{r}126.4 \\
9\end{array}$ \\
\hline 2 & Acacia macracantha Humb. \& Bonpl. ex Willd. & LEGUMINOSAE & 24 & 8.57 & 8 & 9.88 & 0.11556 & 2.68 & 21.12 \\
\hline
\end{tabular}




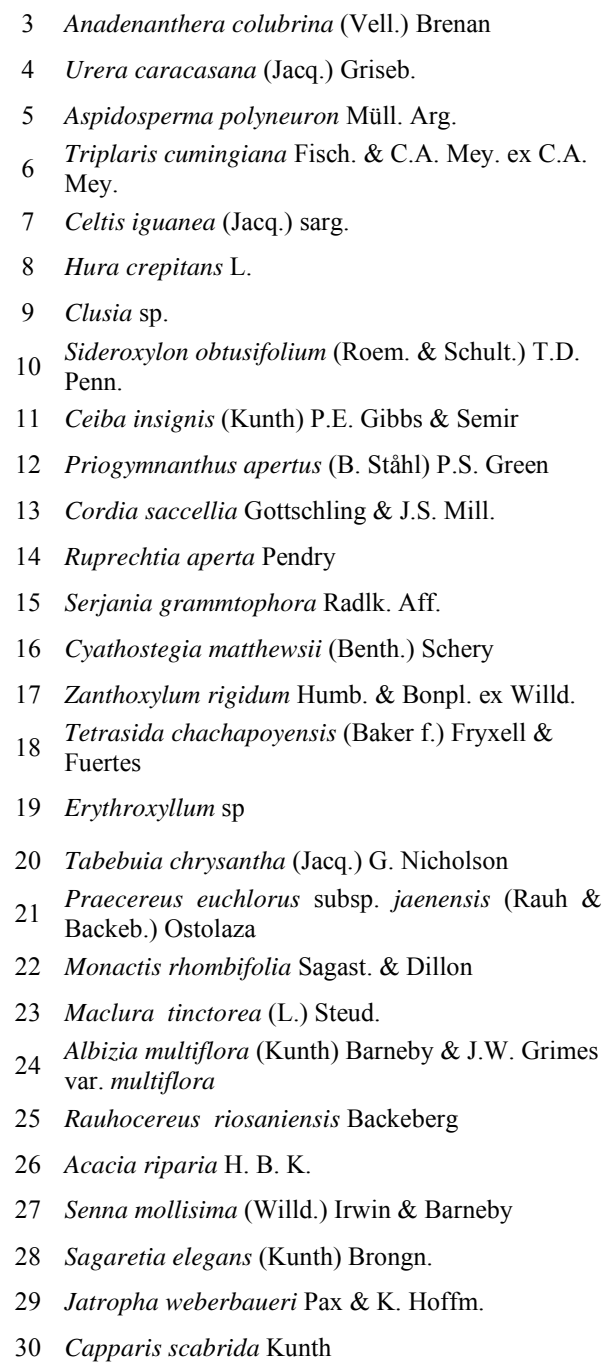

\begin{tabular}{|c|c|c|c|c|c|c|c|}
\hline LEGUMINOSAE & 12 & 4.29 & 5 & 6.17 & 0.32622 & 7.55 & 18.01 \\
\hline URTICACEAE & 17 & 6.07 & 7 & 8.64 & 0.02732 & 0.63 & 15.35 \\
\hline APOCYNACEAE & 9 & 3.21 & 4 & 4.94 & 0.23948 & 5.55 & 13.70 \\
\hline POLYGONACEAE & 8 & 2.86 & 4 & 4.94 & 0.04183 & 0.97 & 8.76 \\
\hline ULMACEAE & 7 & 2.50 & 3 & 3.70 & 0.07258 & 1.68 & 7.88 \\
\hline EUPHORBIACEAE & 3 & 1.07 & 3 & 3.70 & 0.08684 & 2.01 & 6.79 \\
\hline CLUSIACEAE & 4 & 1.43 & 2 & 2.47 & 0.11760 & 2.72 & 6.62 \\
\hline SAPOTACEAE & 2 & 0.71 & 2 & 2.47 & 0.13773 & 3.19 & 6.37 \\
\hline BOMBACACEAE & 4 & 1.43 & 3 & 3.70 & 0.04997 & 1.16 & 6.29 \\
\hline OLEACEAE & 3 & 1.07 & 3 & 3.70 & 0.06141 & 1.42 & 6.20 \\
\hline BORAGINACEAE & 2 & 0.71 & 1 & 1.23 & 0.15242 & 3.53 & 5.48 \\
\hline POLYGONACEAE & 2 & 0.71 & 2 & 2.47 & 0.09749 & 2.26 & 5.44 \\
\hline SAPINDACEAE & 3 & 1.07 & 3 & 3.70 & 0.00128 & 0.03 & 4.80 \\
\hline LEGUMINOSAE & 4 & 1.43 & 2 & 2.47 & 0.00523 & 0.12 & 4.02 \\
\hline RUTACEAE & 3 & 1.07 & 2 & 2.47 & 0.02053 & 0.48 & 4.02 \\
\hline MALVACEAE & 3 & 1.07 & 2 & 2.47 & 0.01331 & 0.31 & 3.85 \\
\hline $\begin{array}{l}\text { ERYTHROXYLACEA } \\
\text { E }\end{array}$ & 3 & 1.07 & 2 & 2.47 & 0.00187 & 0.04 & 3.58 \\
\hline BIGNONIACEAE & 1 & 0.36 & 1 & 1.23 & 0.07306 & 1.69 & 3.28 \\
\hline CACTACEAE & 2 & 0.71 & 2 & 2.47 & 0.00251 & 0.06 & 3.24 \\
\hline ASTERACEAE & 2 & 0.71 & 2 & 2.47 & 0.00111 & 0.03 & 3.21 \\
\hline MORACEAE & 1 & 0.36 & 1 & 1.23 & 0.05187 & 1.20 & 2.79 \\
\hline LEGUMINOSAE & 2 & 0.71 & 1 & 1.23 & 0.02889 & 0.67 & 2.62 \\
\hline CACTACEAE & 2 & 0.71 & 1 & 1.23 & 0.00393 & 0.09 & 2.04 \\
\hline LEGUMINOSAE & 1 & 0.36 & 1 & 1.23 & 0.00108 & 0.02 & 1.62 \\
\hline LEGUMINOSAE & 1 & 0.36 & 1 & 1.23 & 0.00071 & 0.02 & 1.61 \\
\hline RHAMNACEAE & 1 & 0.36 & 1 & 1.23 & 0.00049 & 0.01 & 1.60 \\
\hline EUPHORBIACEAE & 1 & 0.36 & 1 & 1.23 & 0.00049 & 0.01 & 1.60 \\
\hline \multirow[t]{2}{*}{ CAPPARIDACEAE } & 1 & 0.36 & 1 & 1.23 & 0.00049 & 0.01 & 1.60 \\
\hline & 280 & & 81 & & 4.318 & & \\
\hline
\end{tabular}

$\mathrm{AB} \mathrm{A}=$ Abundancia absoluta; $\mathrm{AB} \mathrm{R}=$ Abundancia relativa; FR A = Frecuencia absoluta; FR R = Frecuencia relativa; $\mathrm{DO} A=\mathrm{Dominancia}$ absoluta; DO R = Dominancia relativa; IVI = Índice de valor de importancia.

ANEXO 2. Datos fitosociológicos de 10 transectos de $50 \times 2 \mathrm{~m}$ ( 0.1 ha) levantados en el sector San Isidro, al noreste de la ciudad de Jaén.

\begin{tabular}{lllrrrrrrr}
\hline \multirow{2}{*}{$\mathbf{N}^{\mathbf{0}}$} & \multicolumn{1}{c}{ ESPECIE } & FAMILIA & $\begin{array}{c}\text { AB } \\
\text { A }\end{array}$ & $\begin{array}{c}\text { AB } \\
\text { R }\end{array}$ & $\begin{array}{c}\text { FR } \\
\text { A }\end{array}$ & $\begin{array}{c}\text { FR } \\
\text { R }\end{array}$ & $\begin{array}{c}\text { DO A } \\
\text { DO R }\end{array}$ & IVI \\
\hline \multirow{2}{*}{$\begin{array}{l}\text { Tetrasida chachapoyensis (Baker f.) Fryxell \& } \\
\text { Fuertes }\end{array}$} & MALVACEAE & 52 & 20.2 & 10 & 10.5 & 0.14268 & 6.65478 & 37.41 \\
2 & Cordia iguaguana Melch. ex I.M. Johnst. & BORAGINACEAE & 24 & 9.34 & 4 & 4.21 & 0.39405 & 18.3785 & 31.93 \\
3 & Priogymnanthus apertus (B. Ståhl) P.S. Green & OLEACEAE & 15 & 5.84 & 4 & 4.21 & 0.38932 & 18.1579 & 28.21 \\
4 & Browningia altissima (F. Ritter) Buxb. & CACTACEAE & 16 & 6.23 & 8 & 8.42 & 0.27453 & 12,8042 & 27.45 \\
5 & Capparis flexuosa (L.) L. & CAPPARIDACEAE & 11 & 4.28 & 5 & 5.26 & 0.34472 & 16.0779 & 25.62 \\
6 & Ruprechtia aperta Pendry & POLYGONACEAE & 22 & 8.56 & 9 & 9.47 & 0.07523 & 3.50855 & 21.54 \\
7 & Cyathostegia matthewsii (Benth.) Schery & LEGUMINOSAE & 17 & 6.61 & 6 & 6.32 & 0.01511 & 0.70479 & 13.64 \\
8 & Acacia macracantha Humb. \& Bonpl. ex Willd. & LEGUMINOSAE & 6 & 2.33 & 3 & 3.16 & 0.10120 & 4.7198 & 10.21 \\
9 & Rauhocereus riosanensis Backeberg & CACTACEAE & 12 & 4.67 & 3 & 3.16 & 0.02774 & 1.29367 & 9.12 \\
10 & Croton thurifer Kunth & EUPHORBIACEAE & 10 & 3.89 & 4 & 4.21 & 0.01208 & 0.56339 & 8.66
\end{tabular}




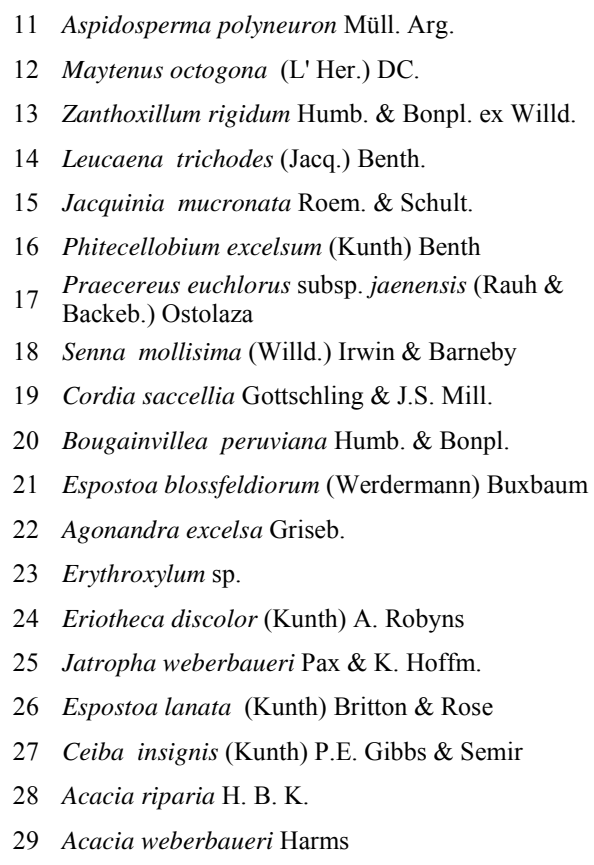

\begin{tabular}{lrrrrrrr} 
APOCYNACEAE & 4 & 1.56 & 4 & 4.21 & 0.05979 & 2.78856 & 8.56 \\
CELASTRACEAE & 11 & 4.28 & 3 & 3.16 & 0.01144 & 0.53339 & 7.97 \\
RUTACEAE & 6 & 2.33 & 4 & 4.21 & 0.02938 & 1.37042 & 7.92 \\
LEGUMINOSAE & 7 & 2.72 & 4 & 4.21 & 0.00922 & 0.42994 & 7.36 \\
THEOPHRASTACEAE & 5 & 1.95 & 3 & 3.16 & 0.04762 & 2.22096 & 7.32 \\
LEGUMINOSAE & 5 & 1.95 & 3 & 3.16 & 0.03732 & 1.74076 & 6.84 \\
CACTACEAE & 7 & 2.72 & 2 & 2.11 & 0.00910 & 0.42441 & 5.25 \\
LEGUMINOSAE & 1 & 0.39 & 1 & 1.05 & 0.07258 & 3.38532 & 4.83 \\
BORAGINACEAE & 6 & 2.33 & 2 & 2.11 & 0.00635 & 0.29594 & 4.74 \\
NYCTAGINACEAE & 2 & 0.78 & 2 & 2.11 & 0.03518 & 1.64087 & 4.52 \\
CACTACEAE & 5 & 1.95 & 1 & 1.05 & 0.00765 & 0.35668 & 3.35 \\
OPILIACEAE & 2 & 0.78 & 2 & 2.11 & 0.00159 & 0.07418 & 2.96 \\
ERYTHROXYLACEAE & 2 & 0.78 & 2 & 2.11 & 0.00102 & 0.04766 & 2.93 \\
BOMBACACEAE & 2 & 0.78 & 1 & 1.05 & 0.02096 & 0.97765 & 2.81 \\
EUPHORBIACEAE & 3 & 1.17 & 1 & 1.05 & 0.00262 & 0.12235 & 2.34 \\
CACTACEAE & 1 & 0.39 & 1 & 1.05 & 0.01327 & 0.61907 & 2.06 \\
BOMBACACEAE & 1 & 0.39 & 1 & 1.05 & 0.00113 & 0.0529 & 1.49 \\
LEGUMINOSAE & 1 & 0.39 & 1 & 1.05 & 0.00062 & 0.02872 & 1.47 \\
LEGUMINOSAE & 1 & 0.39 & 1 & 1.05 & 0.00057 & 0.0267 & 1.47 \\
& 257 & & 95 & & 2.14406 & & 300.00 \\
\hline & & & & & & &
\end{tabular}

ANEXO 3. Datos fitosociológicos de 10 transectos de 50x2 m (0,1 ha) levantados en el sector Shanango, al norte de la ciudad de Jaén, cerca a las ladrilleras.

\begin{tabular}{|c|c|c|c|c|c|c|c|c|c|}
\hline $\mathbf{N}^{\circ}$ & ESPECIE & FAMILIA & $\begin{array}{c}\mathbf{A B} \\
\mathbf{A}\end{array}$ & AB R & $\begin{array}{c}\mathbf{F R} \\
\mathbf{A}\end{array}$ & $\begin{array}{c}\mathbf{F R} \\
\mathbf{R} \\
\end{array}$ & DO A & DO R & IVI \\
\hline 1 & Cordia iguaguana Melch. ex I.M. Johnst. & BORAGINACEAE & 85 & 21.465 & 9 & 8.824 & 0.32236 & 22.336 & 52.625 \\
\hline 2 & Esenbeckia cornuta Eng. & RUTACEAE & 76 & 19.192 & 7 & 6.863 & 0.15469 & 10.719 & 36.774 \\
\hline 3 & $\begin{array}{l}\text { Tetrasida chachapoyensis (Baker f.) Fryxell \& } \\
\text { Fuertes }\end{array}$ & MALVACEAE & 49 & 12.374 & 9 & 8.824 & 0.06145 & 4.258 & 25.455 \\
\hline 4 & $\begin{array}{l}\text { Praecereus euchlorus subsp. jaenensis (Rauh } \\
\text { \& Backeb.) Ostolaza }\end{array}$ & CACTACEAE & 40 & 10.101 & 8 & 7.843 & 0.04281 & 2.966 & 20.910 \\
\hline 5 & Ceiba insignis (Kunth) P.E. Gibbs \& Semir & BOMBACACEAE & 3 & 0.758 & 3 & 2.941 & 0.23073 & 15.987 & 19.686 \\
\hline 6 & Priogymnanthus apertus (B. Ståhl) P.S. Green & OLEACEAE & 18 & 4.545 & 4 & 3.922 & 0.12263 & 8.497 & 16.964 \\
\hline 7 & Ruprechtia aperta Pendry & POLYGONACEAE & 27 & 6.818 & 8 & 7.843 & 0.03250 & 2.252 & 16.913 \\
\hline 8 & Croton thurifer Kunth & EUPHORBIACEAE & 21 & 5.303 & 7 & 6.863 & 0.02158 & 1.496 & 13.661 \\
\hline 9 & Capparis flexuosa (L.) L. & CAPPARIDACEAE & 8 & 2.020 & 6 & 5.882 & 0.07990 & 5.536 & 13.439 \\
\hline 10 & Browningia altissima (F. Ritter) Buxb. & CACTACEAE & 6 & 1.515 & 5 & 4.902 & 0.09718 & 6.733 & 13.150 \\
\hline 11 & Eriotheca discolor (Kunth) A. Robyns & BOMBACACEAE & 4 & 1.010 & 4 & 3.922 & 0.11137 & 7.717 & 12.649 \\
\hline 12 & Cyathostegia matthewsii (Benth.) Schery & LEGUMINOSAE & 13 & 3.283 & 6 & 5.882 & 0.00927 & 0.642 & 9.807 \\
\hline 13 & Rauhocereus riosaniensis Backeberg & CACTACEAE & 11 & 2.778 & 3 & 2.941 & 0.01897 & 1.314 & 7.033 \\
\hline 14 & $\begin{array}{l}\text { Acacia macracantha Humb. \& Bonpl. ex } \\
\text { Willd. }\end{array}$ & LEGUMINOSAE & 5 & 1.263 & 4 & 3.922 & 0.02013 & 1.395 & 6.579 \\
\hline 15 & Jacquinia mucronata Roem. \& Schult. & THEOPHRASTACEAE & 4 & 1.010 & 3 & 2.941 & 0.03753 & 2.601 & 6.552 \\
\hline 16 & $\begin{array}{l}\text { Zanthoxillum rigidum Humb. \& Bonpl. ex } \\
\text { Willd. }\end{array}$ & RUTACEAE & 5 & 1.263 & 4 & 3.922 & 0.00827 & 0.573 & 5.758 \\
\hline 17 & Espostoa lanata (Kunth) Britton \& Rose & CACTACEAE & 1 & 0.253 & 1 & 0.98 & 0.0415477 & 2.879 & 4.112 \\
\hline 18 & Tabebuia chrysantha (Jacq.) G. Nicholson & BIGNONIACEAE & 6 & 1.515 & 2 & 1.961 & 0.00609 & 0.422 & 3.898 \\
\hline 19 & Jatropha weberbaueri Pax \& K. Hoffm. & EUPHORBIACEAE & 4 & 1.010 & 2 & 1.961 & 0.00623 & 0.432 & 3.403 \\
\hline 20 & Pereskia horrida (Kunth) DC. & CACTACEAE & 2 & 0.505 & 2 & 1.961 & 0.00200 & 0.139 & 2.604 \\
\hline 21 & $\begin{array}{l}\text { Sideroxylon obtusifolium (Roem. \& Schult.) } \\
\text { T.D. Penn. }\end{array}$ & SAPOTACEAE & 3 & 0.758 & 1 & 0.98 & 0.00751 & 0.520 & 2.258 \\
\hline 22 & Caesalpinia sp. nova & LEGUMINOSAE & 2 & 0.505 & 1 & 0.98 & 0.00393 & 0.272 & 1.758 \\
\hline
\end{tabular}


DIVERSIDAD Y ENDEMISMOS DE LOS BOSQUES ESTACIONALMENTE SECOS DE JAÉN Diciembre 2007

\begin{tabular}{|c|c|c|c|c|c|c|c|c|c|}
\hline 23 & Heteropsis sp & MALPIGHIACEAE & 1 & 0.253 & 1 & 0.98 & 0.0021237 & 0.147 & 1.380 \\
\hline 24 & Phitecellobium excelsum (Kunth) Benth & LEGUMINOSAE & 1 & 0.253 & 1 & 0.98 & 0.00159 & 0.110 & 1.343 \\
\hline \multirow[t]{2}{*}{25} & Acacia weberbaueri Harms & LEGUMINOSAE & 1 & 0.253 & 1 & 098 & 0.00080 & 0.056 & 1.289 \\
\hline & & TOTAL & 396 & & 102 & & 1.44320 & & 300.00 \\
\hline
\end{tabular}

ANEXO 4. Datos fitosociológicos de 10 transectos de 50x2 m (0.1 ha) levantados en el sector Mochenta, km 3+900 de la carretera Chamaya a Jaén.

\begin{tabular}{|c|c|c|c|c|c|c|c|c|c|}
\hline $\mathbf{N}^{\circ}$ & ESPECIE & FAMILIA & AB A & $\begin{array}{c}\mathbf{A B} \\
\mathbf{R}\end{array}$ & $\begin{array}{c}\mathbf{F R} \\
\mathbf{A}\end{array}$ & $\begin{array}{c}\text { FR } \\
\mathbf{R}\end{array}$ & DO A & DO R & IVI \\
\hline 1 & Browningia altissima (F. Ritter) Buxb. & CACTACEAE & 54 & 19.4 & 9 & 9.89 & 4.17730 & 35,1755 & 64.49 \\
\hline 3 & Anadenanthera colubrina (Vell.) Brenan & LEGUMINOSAE & 26 & 9.35 & 7 & 7.69 & 1.31170 & 11,0453 & 28.09 \\
\hline 4 & Croton thurifer Kunth & EUPHORBIACEAE & 44 & 15.8 & 9 & 9.89 & 0.26244 & 2,20995 & 27.93 \\
\hline 6 & Cyathostegia matthewsii (Benth.) Schery & LEGUMINOSAE & 27 & 9.71 & 9 & 9.89 & 0.24234 & 2,04069 & 21.64 \\
\hline 7 & Acacia weberbaueri Harms & LEGUMINOSAE & 28 & 10.1 & 8 & 8.79 & 0.20144 & 1,69623 & 20.56 \\
\hline 8 & $\begin{array}{l}\text { Sideroxylon obtusifolium (Roem. \& Schult.) } \\
\text { T.D. Penn. }\end{array}$ & SAPOTACEAE & 7 & 2.52 & 4 & 4.4 & 0.48599 & 4,09234 & 11.01 \\
\hline 9 & Jacquinia mucronata Roem. \& Schult. & THEOPHRASTACEAE & 6 & 2.16 & 4 & 4.4 & 0.17053 & 1,43593 & 7.99 \\
\hline 12 & Pereskia horrida (Kunth) DC. & CACTACEAE & 7 & 2.52 & 3 & 3.3 & 0.00542 & 0,04561 & 5.86 \\
\hline 13 & Ruprechtia aperta Pendry & POLYGONACEAE & 6 & 2.16 & 2 & 2.2 & 0.09047 & 0,76183 & 5.12 \\
\hline 14 & Jatropha weberbaueri Pax \& K. Hoffm. & EUPHORBIACEAE & 4 & 1.44 & 2 & 2.2 & 0.05622 & 0,47338 & 4.11 \\
\hline 15 & Agonandra excelsa Griseb. & OPILIACEAE & 3 & 1.08 & 2 & 2.2 & 0.00757 & 0,06376 & 3.34 \\
\hline 16 & $\begin{array}{l}\text { Praecereus euchlorus subsp. jaenensis (Rauh } \\
\text { \& Backeb.) Ostolaza }\end{array}$ & CACTACEAE & 4 & 1.44 & 1 & 1.1 & 0.07118 & 0,59935 & 3.14 \\
\hline 17 & Bougainvillea peruviana Humb. \& Bonpl. & NYCTAGINACEAE & 2 & 0.72 & 2 & 2.2 & 0.01100 & 0,09262 & 3.01 \\
\hline 18 & Espostoa mirabilis F. Ritter & CACTACEAE & 1 & 0.36 & 1 & 1.1 & 0.10810 & 0,9103 & 2.37 \\
\hline 19 & Cordia saccellia Gottschling \& J.S. Mill. & BORAGINACEAE & 2 & 0.72 & 1 & 1.1 & 0.02460 & 0,20711 & 2.03 \\
\hline 25 & Espostoa superba F. Ritter & CACTACEAE & 1 & 0.36 & 1 & 1.1 & 0.00866 & 0,07291 & 1.53 \\
\hline 26 & Aloysias scorodonioides (Kunth) Cham. & VERBENACEAE & 1 & 0.36 & 1 & 1.1 & 0.00503 & 0,04233 & 1.50 \\
\hline 27 & $\begin{array}{l}\text { Tetrasida chachapoyensis (Baker f.) Fryxell } \\
\text { \& Fuertes }\end{array}$ & MALVACEAE & 1 & 0.36 & 1 & 1.1 & 0.00132 & 0,01112 & 1.47 \\
\hline \multirow[t]{2}{*}{28} & Bauhinia acuelata L. & LEGUMINOSAE & 1 & 0.36 & 1 & 1.1 & 0.00080 & 0,00677 & 1.47 \\
\hline & & & 278.00 & & 91 & & 11.87559 & & 300.00 \\
\hline
\end{tabular}

\footnotetext{
${ }^{1}$ Herbario MOL. Facultad de Ciencias Forestales. Universidad Nacional Agraria La Molina. Lima Perú. jlmarcelop@lamolina.edu.pe ${ }^{2}$ Herbario MOL. Facultad de Ciencias Forestales. Universidad Nacional Agraria La Molina. Lima Perú. reynel@lamolina.edu.pe

${ }^{3}$ Herbario MOL. Facultad de Ciencias Forestales. Universidad Nacional Agraria La Molina. Lima Perú. pazpolli@lamolina.edu.pe

${ }^{4}$ Laboratorio de Silvicultura. Facultad de Ciencias Forestales. Universidad Nacional Agraria La Molina. Lima Perú. fbulnes@lamolina.edu.pe

${ }^{5}$ Herbario MOL. Facultad de Ciencias Forestales. Universidad Nacional Agraria La Molina. Lima Perú. 20030264@lamolina.edu.pe
} 